\title{
Impacts of black carbon and mineral dust on radiative forcing and glacier melting during summer in the Qilian Mountains, northeastern Tibetan Plateau
}

Yang $\mathrm{Li}^{1,5^{*}}$, Jizu Chen ${ }^{2,5}$, Shichang Kang ${ }^{2,3^{*}}$, Chaoliu $\mathrm{Li}^{1}$, Bin $\mathrm{Qu}^{4}$, Lekhendra Tripathee ${ }^{1,5}$, Fangping Yan ${ }^{2,5}$, Yulan Zhang ${ }^{2}, \mathrm{Junming} \mathrm{Guo}^{1,5}$,

Chaman $\mathrm{Gul}^{2,5}$, Xiang Qin ${ }^{2}$

${ }^{1}$ Key Laboratory of Tibetan Environment Changes and Land Surface Processes, Institute of Tibetan Plateau Research, Chinese Academy of Sciences, Beijing, 100101, China. ${ }^{2}$ State Key Laboratory of Cryospheric Sciences, Cold and Arid Regions Environmental and Engineering Research Institute, Chinese Academy of Sciences, Lanzhou, 730000 , China. ${ }^{3}$ CAS Center for Excellence in Tibetan Plateau Earth Sciences, Beijing 100085, China.

${ }^{4}$ Laboratory of Green Chemistry, Lappeenranta University of Technology, Mikkeli 50130, Finland.

${ }^{5}$ University of Chinese Academy of Sciences, Beijing 100049, China.

Correspondence to: Y. Li (kerwinli@126.com) and S.-C, Kang (shichang.kang@1zb.ac.cn)

Table S1. Parameters for sensitivity analysis with SNICAR. 1. Elevation (m a.s.l); 2. Solar zenith angle; 3. Snowpack density (kg m-3); 4. Snowpack thickness (cm); 5. Snow grain effective radius $(\mu \mathrm{m}) ; 6$. BC concentration ( $\mathrm{ng} \mathrm{g}^{-1}$, Surface-coated); 7. MD concentration $\left(\mu \mathrm{g} \mathrm{g}^{-1}\right) ; 8$. Simulated albedo of snow including both BC and MD; 9 . Simulated albedo of snow including BC only; 10. Simulated albedo of pure snow; 11 and 12 are the contributions of BC and MD to the total reduction of the albedo (\%), respectively.13. RF by MD; 14. RF by BC;

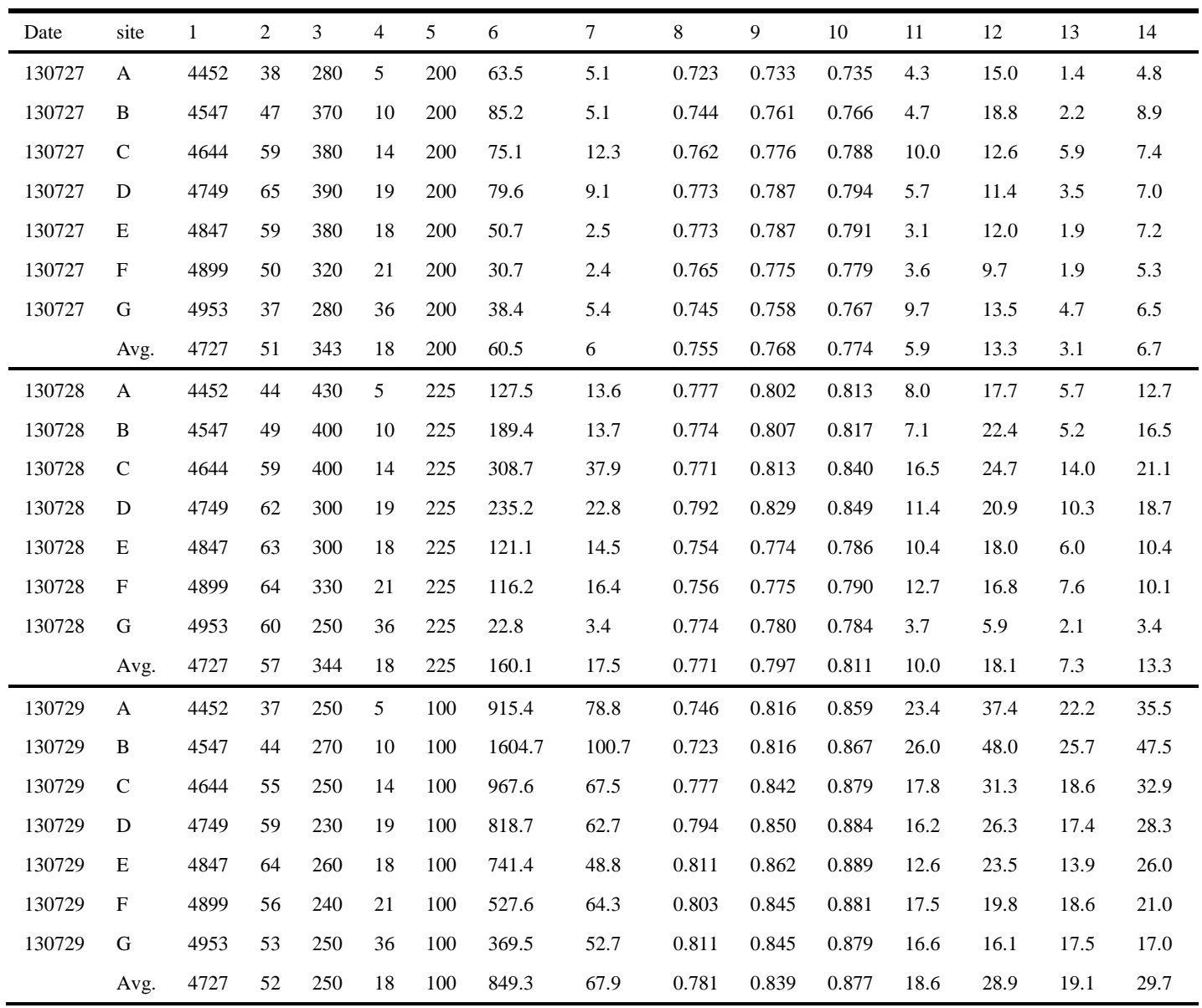









\begin{tabular}{|c|c|c|c|c|c|c|c|c|c|c|c|c|c|c|c|}
\hline 140806 & $\mathrm{C} 1$ & 4501 & 55 & 400 & 1 & 400 & 22424.7 & 2360 & 0.259 & 0.448 & 0.560 & 36.5 & 61.2 & 60.8 & 101.9 \\
\hline 140806 & $\mathrm{C} 2$ & 4501 & 55 & 400 & 1 & 400 & 22011.6 & 2465 & 0.261 & 0.444 & 0.560 & 37.5 & 59.7 & 62.5 & 99.4 \\
\hline 140806 & $\mathrm{C} 3$ & 4549 & 59 & 400 & 1 & 400 & 40265.8 & 3300 & 0.229 & 0.440 & 0.576 & 42.1 & 65.0 & 73.8 & 113.9 \\
\hline 140806 & D1 & 4549 & 59 & 400 & 1 & 400 & 45310.6 & 5232 & 0.224 & 0.404 & 0.576 & 53.1 & 55.5 & 93.1 & 97.3 \\
\hline 140806 & D2 & 4549 & 59 & 400 & 1 & 400 & 48110.3 & 5292 & 0.220 & 0.403 & 0.576 & 53.4 & 56.5 & 93.6 & 99.0 \\
\hline 140806 & E1 & 4600 & 63 & 400 & 1 & 400 & 31203.1 & 3290.3 & 0.267 & 0.458 & 0.593 & 39.5 & 56.2 & 72.8 & 103.6 \\
\hline 140806 & E2 & 4600 & 63 & 400 & 1 & 400 & 3421.2 & 340 & 0.481 & 0.570 & 0.593 & 6.7 & 26.0 & 12.4 & 47.9 \\
\hline 140806 & E3 & 4651 & 65 & 400 & 1 & 400 & 5871.6 & 700 & 0.439 & 0.558 & 0.602 & 12.4 & 34.1 & 23.4 & 64.4 \\
\hline 140806 & $\mathrm{~F} 1$ & 4651 & 65 & 400 & 1 & 400 & 12431.3 & 1450 & 0.362 & 0.524 & 0.602 & 22.3 & 46.4 & 42.1 & 87.8 \\
\hline 140806 & G1 & 4699 & 67 & 400 & 1 & 400 & 9603.8 & 1075 & 0.400 & 0.549 & 0.611 & 17.1 & 41.7 & 33.1 & 81.0 \\
\hline 140806 & $\mathrm{G} 2$ & 4699 & 67 & 400 & 1 & 400 & 6242.3 & 910 & 0.440 & 0.557 & 0.611 & 14.9 & 32.6 & 28.9 & 63.3 \\
\hline 140806 & G3 & 4699 & 67 & 400 & 1 & 400 & 6829.8 & 1175 & 0.428 & 0.545 & 0.611 & 18.3 & 32.6 & 35.5 & 63.2 \\
\hline 140806 & $\mathrm{H} 1$ & 4750 & 67 & 400 & 1 & 400 & 22013.6 & 2446.4 & 0.317 & 0.500 & 0.611 & 30.8 & 51.1 & 59.8 & 99.2 \\
\hline 140806 & $\mathrm{H} 2$ & 4750 & 67 & 400 & 1 & 400 & 30089.4 & 3425.8 & 0.290 & 0.475 & 0.611 & 37.7 & 51.7 & 73.2 & 100.2 \\
\hline 140806 & $\mathrm{H} 3$ & 4750 & 67 & 400 & 1 & 400 & 17450.3 & 2173.1 & 0.338 & 0.508 & 0.611 & 28.6 & 47.5 & 55.4 & 92.2 \\
\hline 140806 & $\mathrm{H} 4$ & 4802 & 66 & 400 & 1 & 400 & 44764.1 & 4985.7 & 0.262 & 0.447 & 0.606 & 45.0 & 52.4 & 86.1 & 100.3 \\
\hline 140806 & I1 & 4802 & 66 & 400 & 1 & 400 & 18641.4 & 2043.8 & 0.332 & 0.512 & 0.606 & 26.5 & 50.9 & 50.7 & 97.4 \\
\hline \multirow[t]{2}{*}{140806} & $\mathrm{~K} 3$ & 4904 & 60 & 400 & 1 & 400 & 3526.8 & 526.1 & 0.459 & 0.546 & 0.580 & 10.5 & 26.4 & 18.6 & 46.8 \\
\hline & Avg. & 4668 & 62 & 400 & 1 & 400 & 22704.1 & 2619 & 0.331 & 0.486 & 0.590 & 31.6 & 46.3 & 56.2 & 84.0 \\
\hline
\end{tabular}

